\title{
Learning from excellence in healthcare: a new approach to incident reporting
}

\author{
Nicola Kelly, ${ }^{1}$ Simon Blake, ${ }^{1,2}$ Adrian Plunkett ${ }^{1}$
}

Tell me how you measure me, and I will tell you how I will behave.

\section{Eliyahu Moshe Goldratt ${ }^{1}$}

\section{EXCELLENCE IN HEALTHCARE SAFETY}

The pursuit of patient safety is a key component of the wider endeavour to improve quality of healthcare delivery. In its contemporary form, safety is characterised as a condition where the occurrence of adverse outcomes is minimised. Our efforts to improve safety are therefore almost entirely focused on identifying adverse incidents and errors, and implementing adaptations to avoid their recurrence. This reactive approach to safety ('Safety-I') advocates incident reporting and root-cause analysis in order to identify adverse incidents and their causes. ${ }^{2}$ Following major reports emphasising the significant role of human error ${ }^{3}$ and the need for organisational learning from adverse incidents, ${ }^{4}$ incident reporting has become well established in the National Health Service (NHS). Trends of monitoring and reporting of adverse events are increasing. 56

While this approach may achieve good results, with higher levels of adverse incident reporting correlating with a more positive safety culture, ${ }^{5}$ some studies of healthcare safety interventions suggest that the benefits of this approach are limited. ${ }^{7} 8$ Adverse incident reporting is widely encouraged in the NHS, but reporting rates and methods for investigating incidents vary widely between organisations. Staff and patients may not always receive feedback about incidents in which they have been involved, and there is often inadequate evidence of lessons learned or effective change implemented following incidents. 9

\section{SECOND VICTIMS}

An important consideration of Safety-I practice is the potential negative impact on healthcare workers. Staff involved in incidents may often experience the

${ }^{1} \mathrm{PICU}$, Birmingham Children's Hospital NHS Foundation Trust, Birmingham, UK; ${ }^{2}$ Warwick Business School, Coventry, UK

Correspondence to Dr Adrian Plunkett, PICU, Birmingham Children's Hospital NHS Foundation Trust, Birmingham B4 6NH, UK; adrian.plunkett@bch.nhs.uk second-victim phenomenon. ${ }^{10}$ Effects on second victims may include detachment, anxiety and depression, as well as reduced clinical confidence and cognitive functioning, potentially impairing that individual's clinical performance. Some may go on to suffer long-standing issues, similar to posttraumatic stress disorder, experiencing flashbacks, sleep disturbance and situational avoidance, provoking some to leave their job or commit suicide. ${ }^{11} 12$ This phenomenon may be exacerbated if professionals experience a punitive approach towards the reporting and investigation of an error. ${ }^{13}{ }^{14}$ Staff perceptions that disciplinary action or workplace discrimination may take place as a result of reporting an incident can also reduce future engagement in incident reporting. $^{15}$

\section{ELIMINATE THE NEGATIVE}

In healthcare, we tend to place greater emphasis on identifying and examining failures of systems or individuals rather than recognising and reflecting on positive processes or outcomes. However, adverse incidents only account for the minority of healthcare interactions. ${ }^{16}$ It is therefore probable that, by overlooking episodes of high-quality practice, we are missing key opportunities to learn and develop and to be creative and innovative. Why do we have such a bias towards negativity?

Psychologists have long recognised that humans have an innate negativity bias; that we are more attentive to and influenced by negative events, emotions and interpersonal interactions than by the positive. $^{17} 18$ In a recent social experiment, subjects were more likely to choose to read a negative news story than a positive one, and the preponderance of bad news in our daily headlines only serves to support the theory that bad news sells. ${ }^{19}$ Negativity bias has been well described within the field of economics, with Kahneman's work on Prospect theory demonstrating that humans value loss more than the equivalent amount of gain; we like to win but we hate to lose. ${ }^{20}$ This phenomenon can also be seen in our approach to medical education and training, where simulation of 'worst-case' situations and a focus on our shortcomings within revalidation and appraisal may further cultivate a negativity bias within healthcare professionals. $^{21}$

However, recent psychological research has revealed that people can learn effectively both from reflecting on failure (negative reinforcement) and success (positive reinforcement). ${ }^{22}$ In fact, animal studies suggest that success and positive experiences have an enhanced positive influence on the brain compared with failure by triggering dopamine surges, thereby improving neural processing and future performance. $^{23}$

Studies involving front-line healthcare professionals have shown that nurturing positivity in individuals and teams is linked with improved resilience and ability to deal with adversity. ${ }^{24}$ There is also a strong relationship between staff morale and patient experience, with NHS patients reporting better experiences of healthcare in settings where their caregivers feel content and are supported within a positive organisational climate. ${ }^{25}$ This is more relevant now than ever as a recent survey of almost 30000 NHS employees has reported low levels of staff well-being and morale, with $66 \%$ considering leaving their current post. ${ }^{26}$

Meanwhile, other industries, including sports, aviation and engineering, are starting to recognise the value of positivity and resilience. $^{27} 28$ The sports industry, for example, have realised that they are crucial for an athlete to excel. ${ }^{29} 30$ Many practitioners apply a positive psychological model of core competencies, which are crucial to resilience. One such competency is optimism: noticing the good. ${ }^{27}$

\section{ACCENTUATE THE POSITIVE}

In healthcare, there is innate performance variability within almost all systems, which explains why things sometimes go wrong, but more often why things go well. ${ }^{30}$ It is often true that in medicine patients are seldom 'textbook' cases and our working environment is rarely perfect, but rather it is risky, unpredictable and challenging. Human performance varies in response to the changing environment. While some of this variation is unsuccessful, leading to error or harm, a great deal of variation in performance leads to success through adaptive adjustment. These adjustments, or 'workarounds', are strategies for overcoming problems or limitations to compensate for variable conditions. $^{2}$ This is an example of resilience in the healthcare context, which allows an individual or team to function effectively in a demanding and changeable environment. Although a clinical outcome on such an occasion may not 
be outstanding or perfect, the adaptability or resilience employed by staff in order to ensure a safe and satisfactory outcome could in itself be viewed as excellent practice.

Noticing everyday examples of good practice, and learning from the adjustments required for successful outcomes in variable conditions, 'Safety-II', is an emerging approach to safety. Safety-II originates from the concept of resilience engineering, a new way of considering workplace performance and safety, taking into account 'work as done' versus 'work as imagined'. ${ }^{31}$ Resilience in this context refers to the ability of a system to adjust its functioning in response to changes in conditions. The goal of the Safety-II approach is to ensure as many successful outcomes as possible by recognising and learning from good practice and functional adaptations to variation in conditions. Resilience engineering and Safety-II strategies are being used in other high-risk industries such as air traffic management. The Resilience in Healthcare Initiative, led by Professor Erik Hollnagel, aims to apply resilience engineering practice to healthcare worldwide using Safety-II methodology as a key foundation. ${ }^{2}$

A related, emerging approach in healthcare is the 'positive deviance' model, which looks at the variations in performance and process that result in good outcomes rather than harm. The hypothesis behind this model is that by seeking and studying groups or individuals who perform exceptionally well, methods for best practice can be identified and disseminated to improve wider performance. ${ }^{30}$ Safety-II and positive deviance methodologies are not currently highly prevalent in healthcare. Reasons for this may include the lack of clear strategies for defining and measuring excellent and safe practice; our innate negativity bias; or our current regulatory climate, which focuses our attention and resources on harm. We believe that improving resilience in healthcare organisations, through application of concepts such as Safety-II and positive deviance, will benefit patients and staff by optimising safety and by helping to improve service quality and efficacy.

\section{LEARNING FROM EXCELLENCE}

We have developed a new initiative, Learning from Excellence (LfE), which aims to provide a means of identifying and capturing learning from episodes of peer-reported excellence or positive deviance. While adverse incident reporting has been inspired by the Japanese edict quoted by Berwick ${ }^{32}$ 'every defect is a treasure', the spirit of LfE is to treasure and appreciate our everyday successes, rather than taking them for granted. LfE was developed with the hypothesis that reporting and studying success would augment learning, enhance patient outcomes and experience through quality improvement work and positively impact resilience and culture in the workplace.

LfE began with a pilot project in Birmingham Children's Hospital's Paediatric Intensive Care department (PICU) as a system for all staff to voluntarily report episodes of excellent practice. Reports are submitted via an intranetbased 'IR2' form, suitably juxtaposed to the adverse incident reporting 'IR1' form used within our organisation. We did not provide guidance or restrictions on which types of episode to report, leaving the reporter to apply their own definition of 'excellence'. Staff in receipt of an IR2 receive an automatic email notification and the reporter receives an email acknowledgement. LfE is championed by a small multidisciplinary team of frontline clinical staff, who review all reports, creating weekly summaries with learning points, which are shared with the whole department through an e-bulletin.

\section{STUDYING EXCELLENCE}

In order to identify and enhance learning opportunities, reports are given an in-depth consideration at an 'IRIS' (Improving Resilience, Inspiring Success) or 'reverse SIRI' (Serious Incident Report Investigation) meeting. An IRIS involves a group dialogue between those submitting and receiving the IR2 of interest; facilitated by LfE team members using appreciative inquiry (AI) methodology. (A glossary of terms is included in see online supplementary appendix 1.) These hour-long informal reviews aim to identify how excellence was achieved, including 'workarounds' or innovations employed, and to generate ideas for sharing and promoting excellence.

AI was selected as a framework for IRIS as it nurtures a positive mind-set and helps gain new insights into moments of optimal performance. The generative dialogic nature of AI encourages participants to share and reflect upon their ideas for positive change. ${ }^{33-36}$ Healthcare leaders advocate using the AI approach for its potential to support organisations with significant performance challenges, and as a key solution to engendering successful change in the NHS. ${ }^{37} 38$

A recent IRIS identified an episode of innovation by a bedside nurse in PICU regarding more efficient delivery of peritoneal dialysis. In addition to highlighting an excellent piece of new practice, the AI discussion led us to consider ideas for how we can better capture innovation and workarounds in front-line healthcare by empowering front-line staff to share ideas using LfE as a tool. We have also started to develop ways of looking at positive feedback from families using AI to glean new insights and ideas for improving patient experience on PICU.

\section{EVALUATING LFE}

Trend analysis of LfE reporting demonstrates rising reporting rates since project launch over a wide range of excellence domains and clinical contexts, suggesting acceptance and proof of concept for LfE. We have received $>700$ reports since the launch of the initiative and apparent 'viral spread' of the concept with rising reporting outside of PICU from a wide variety different professional groups and hospital departments. Figure 1 shows the trend in monthly frequency of reporting.

One year into the pilot, a formal evaluation was undertaken via an anonymous online survey to all PICU staff, including doctors, nurses, allied healthcare professionals, managers and administrators, to determine their perception of how LfE has impacted team learning, patient care and staff morale. A $67 \%$ survey response rate (229/339 PICU staff members) was achieved, with all staff groups in the department represented. Overall, staff feedback demonstrated clear support for the LfE initiative and the philosophy underlying it. Survey responses strongly supported the hypothesis that excellence reporting can improve staff morale (93\% agree or strongly agree) and improve quality of care $(87 \%$ agree or strongly agree). Our staff perceive that learning from studying excellent practice is as valuable as reflecting on individual error, and both are more valuable than studying the failures of others.

Examples of free-text comments include:

...LfE is a great way of improving team morale and celebrating the great work we do in PICU.

I am convinced that if we create a process where our staff are praised more frequently, we will be able to retain more staff. It's as simple as thinking about what makes a day a good day, for me it's receiving a pat on the back and the reassurance I am doing an ok job. 


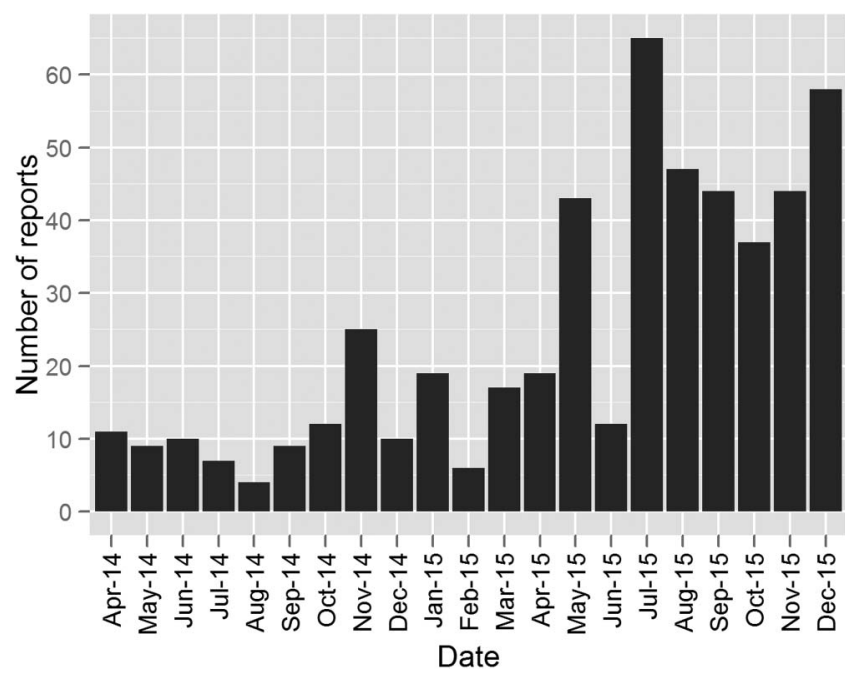

Figure 1 Frequency of excellence reporting.

\section{TANGIBLE RESULTS}

We have started applying the LfE concept to specific areas of quality improvement. Our first prospective pilot study focused on improving the standards of antimicrobial prescription documentation in PICU. Interventions included IR2 reporting of all clinicians who achieved gold-standard prescriptions, anonymous gold-standard prescription displays in prescribing areas, electronic bulletins and face-to-face educational sessions. Rates of gold-standard prescribing increased significantly from $18 \%$ to $35 \%\left(\mathrm{p}=0.045 ; \chi^{2}\right)$ post intervention. Quality improvement and audit projects usually focus on deficit management -that is, reduction of errors. In this project, we have taken a novel approach by demonstrating an improvement at the other end of the quality spectrum: goldstandard ('perfect') prescribing-a metric not usually measured in quality improvement projects. We are now exploring the application of this methodology in other areas of PICU practice (eg, care bundle compliance).

\section{EXCELLENCE: THE FUTURE}

While current safety strategies have contributed to improved patient care, a broader approach, which redresses the balance between analysing failure and success, is worth pursuing. The LfE initiative is proof of concept that excellence reporting can be used as a tool to capture useful workarounds and adaptations at the 'sharp-end'-essential components of the Safety-II approach. The use of AI to investigate excellence has generated new insights into understanding how processes can work optimally, and this methodology (along with related practices, such as cooperative inquiry) should be explored further in the future.

\section{Excellence is a better teacher than medi- ocrity. The lessons of the ordinary are} everywhere.

Truly profound and original insights are to be found only in studying the exemplary. ${ }^{39}$

Beyond the benefits to patient care and staff development, there are additional advantages that could motivate the healthcare industry to study excellence routinely. Along with the conventional practice of highlighting and understanding the root causes of error, an emphasis upon identifying, sharing and modelling excellent practice may reduce harm to second victims. LfE has demonstrated the potential for positive effects on workplace culture and morale. It has also helped our local team to share good practice, identify and support innovation, and to discover new ideas and insights to inspire and facilitate quality improvement, with tangible results in areas such as safe prescribing.

LfE is one possible strategy for implementing excellence reporting. The approach identifies and promotes excellent practice within a front-line clinical environment. However, it could also be applied effectively to a wider range of healthcare settings including medical training, professional development and healthcare governance. While the particular methods used by LfE may not suit all healthcare environments, the basic principles could be adapted and applied to engage individuals and teams in the process of identifying and sharing excellence in everyday practice. This intervention may also help to develop a workplace culture whereby team members feel more appreciated and motivated and their hard work is recognised with positive feedback.

There is scope for discovering additional applications of the LfE concept in clinical practice. For example, we are currently working on ways to involve patients and their families in defining what excellent care means for them and how we can learn from their positive experiences of healthcare. Many colleagues working in different settings across the country have indicated that they share our vision and are developing similar projects. This groundswell of interest is an opportunity to establish collaborative ventures in research and quality improvement to understand the potential impact of this concept. We are keen to hear from other groups and organisations about any ideas or schemes they have, and to share our LfE resources, including evaluation data and templates for IRIS meetings. Resources are freely available online at http://www.learningfromexcellence.com.

Twitter Follow Adrian Plunkett at @adrianplunkett

Acknowledgements The authors would like to thank their excellent colleagues at Birmingham Children's Hospital for their ongoing participation in and support for the LfE project.

Contributors NK collaborated in the LfE project, drafted and revised the paper. AP initiated the collaborative LfE project and revised the draft paper. SB collaborated in the LfE project and revised the paper.

\section{Competing interests None declared.}

Provenance and peer review Commissioned; externally peer reviewed.

- Additional material is published online only. To view please visit the journal online (http://dx.doi.org/10. 1136/archdischild-2015-310021).

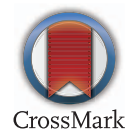

To cite Kelly N, Blake S, Plunkett A. Arch Dis Child 2016;101:788-791.

Received 16 December 2015

Revised 11 April 2016

Accepted 17 April 2016

Published Online First 4 May 2016

Arch Dis Child 2016;101:788-791.

doi:10.1136/archdischild-2015-310021

\section{REFERENCES}

1 Goldratt E. Theory of constraints. Croton-on-Hudson, NY: North River, 1990.

2 Hollnagel E, Wears RL, Braithwaite J. From Safety-I to Safety-II: A White Paper. The Resilient Health Care Net: Published simultaneously by the University of Southern Denmark, University of Florida, USA, and Macquarie University, Australia, 2015.

3 Kohn LT, Corrigan JM, Donaldson MS. To err is human: building a safer health system. Washington: National Academy Press, 2000. 
4 Department of Health. An organisation with a memory, 2000. http://patientsafety.health.org.uk/ resources/organisation-memory (accessed $13 \mathrm{Dec}$ 2015).

5 Hutchinson A, Young TA, Cooper KL, et al. Trends in healthcare incident reporting and relationship to safety and quality data in acute hospitals: results from The National Reporting and Learning System. Qual Saf Health Care 2009;18:5-10.

6 Battles JB and Stevens DP. Adverse event reporting systems and safer healthcare. Qual Saf Health Care 2009:18:2.

7 Landrigan CP, Parry GJ, Bones CB, et al. Temporal Trends in Rates of Patient Harm Resulting from Medical Care. N Engl J Med 2010;363:2124-34.

8 Benning A, Ghaleb M, Suokas A, et al. Large scale organisational intervention to improve patient safety in four UK hospitals: mixed method evaluation. BMJ 2011;342:d195.

9 Wallace L. Feedback from reporting patient safety incidents - are NHS trusts learning lessons? J Health Serv Res Policy 2010;15(Suppl 1):75-8.

10 Wu AW, Steckelberg RC. Medical error, incident investigation and the second victim: doing better but feeling worse? BMJ Qual Saf 2012;21:4.

11 Scott SD, Hirschinger LE, Cox KR, et al. The natural history of recovery for the health care provider "second victim" after adverse patient events. Qual Saf Health Care 2009;18:325-30.

12 Kronman AC, Paasche-Orlow M, Orlander JD. Factors associated with disclosure of medical errors by housestaff. BMJ Qual Saf 2012:21:271-8.

13 Venus E, Galam E, Aubert JP, et al. Medical errors: impact on and management by French general practitioners in training. A study of 70 questionnaires and 10 semi-structured interviews. BMJ Qual Saf 2012;21:279-86.

14 Stewart K, Lawton R, Harrison H. Supporting "second victims" is a system-wide responsibility. BMJ 2015;350:h2341.
15 Mahajan RP. Critical incident reporting and learning. Br J Anaesth 2010;105:69-75.

16 Vincent C, Neale G, Woloshynowych M. Adverse events in British hospitals: preliminary retrospective record review. BMJ 2001;322:517-19.

17 Baumeister RF, Bratslavsky E, Finkenauer C, et al. Bad is stronger than good. Rev Gen Psychol 2001;5:323-70.

18 Rozin P, Royzman EB. Negativity bias, negativity dominance, and contagion. Pers Soc Psychol Rev 2001;5:296-320.

19 Trussler M, Soroka L. Consumer demand for cynical and negative news frames. Int I Press/Polit 2014;19:360-79

20 Kahneman D, Tversky A. Prospect theory: an analysis of decision under risk. Econometrica 1979;47:263.

21 Haizlip J, May N, Schorling J, et al. Perspective: the negativity bias, medical education, and the culture of academic medicine: why culture change is hard. Acad Med 2012:87:1205-9.

22 Ellis S, Carette B, Anseel F, et al. Systematic reflection: implications for learning from failures and successes. Curr Dir Psychol Sci 2014;23:67-72.

23 Histed MH, Pasupathy A, Miller EK. Learning substrates in the primate prefrontal cortex and striatum: sustained activity related to successful actions. Neuron 2009;63:244-53.

24 Jackson D, Firtko A, Edenborough M. Personal resilience as a strategy for surviving and thriving in the face of workplace adversity: a literature review. J Adv Nurs 2007;60:1-9.

25 Maben J, Peccei R, Adams M, et al. Patients experiences of care and the influence of staff motivation, affect and wellbeing. Final report. NIHR Service Delivery and Organisation programme, 2012.

26 Thomson Reuters. NHS staff survey on pay and conditions. 2014. http://www.nhsstaffsurveys.com/ Page/1006/Latest-Results/2014-Results/ (accessed 13 Dec 2015).
27 Gittell JH, Cameron K, Lim S, et al. Relationships, layoffs, and organizational: resilience airline industry responses to September 11. J App/Behav Sci 2006:42:300-29.

28 Seligman ME, Reivich KJ, McBride S. Master resilience training in the U.S. Army. Am Psychol 2011;66:25-34.

29 Sarkar M, Fletcher D. Psychological resilience in sport performers: a review of stressors and protective factors. J Sports Sci 2014;32:1419-34.

30 Lawton R, Taylor N, Clay-Williams R, et al. Positive deviance: a different approach to achieving patient safety. BMJ Qual Saf 2014;23:880-3.

31 Hollnagel E, Woods D, Leveson N. Resilience engineering: concepts and precepts. Ashgate Publishing Ltd., 2007.

32 Berwick DM. Continuous improvement as an ideal in healthcare. N Engl J Med 1989;320:53-6.

33 Shendell-Falik N, Feinson M, Mohr BJ. Enhancing patient safety: improving the patient handoff process through appreciative inquiry. J Nurs $A d m$ 2007:37:95-104.

34 Cooperrider DL, Whitney D. A positive revolution in change: appreciative inquiry. Public Adm Public Policy 2001;87:611-30.

35 Gergen MM, Gergen KJ, Barrett F. Appreciative inquiry as dialogue: generative and transformative. Adv Appreciat Inq 2004;1:3-27.

36 Trajkovski S, Schmied V, Vickers $M$, et al. Implementing the 4D cycle of appreciative inquiry in health care: a methodological review. J Adv Nurs 2013:69:1224-34.

37 Bevan H. 'What Works: Appreciating Our Assets'. 2004. http://www.institute.nhs.uk/quality_and_value/ introduction/article 1.html (accessed 13 Dec 2015)

$38 \mathrm{HSJ} / \mathrm{NT} / \mathrm{NHSiQ}$ Challenge Top-Down Change. 2015. http://m.hsj.co.uk/5083743.article (accessed 13 Dec 2015).

39 Bennis W, Bennis WG. On becoming a leader. Basic Books, 2009. 